\title{
Encoding of Novel Verbal Instructions for Prospective Action in the Lateral Prefrontal Cortex: Evidence from Univariate and Multivariate Functional Magnetic Resonance Imaging Analysis
}

\author{
Nicolas J. Bourguignon ${ }^{1}$, Senne Braem ${ }^{1}$, Egbert Hartstra ${ }^{2}$, \\ Jan De Houwer ${ }^{1}$, and Marcel Brass ${ }^{1}$
}

\begin{abstract}
Verbal instructions are central to humans' capacity to learn new behaviors with minimal training, but the neurocognitive mechanisms involved in verbally instructed behaviors remain puzzling. Recent functional magnetic resonance imaging (fMRI) evidence suggests that the right middle frontal gyrus and dorsal premotor cortex (rMFG-dPMC) supports the translation of symbolic stimulus-response mappings into sensorimotor representations. Here, we set out to (1) replicate this finding, (2) investigate whether this region's involvement is specific to novel (vs. trained) instructions, and (3) study whether rMFG-dPMC also shows differences in its (voxel) pattern response indicative of general cognitive processes of instruction implementation. Participants were shown instructions, which they either had to perform later or merely memorize. Orthogonal to this manipulation, the instructions were
\end{abstract}

\section{INTRODUCTION}

Verbal instructions play a pivotal role in humans' capacity to assimilate new behaviors with minimal practice, avoiding protracted, laborious phases of trial-and-error or imitationbased learning (Morgan et al., 2015; Ramamoorthy \& Verguts, 2012). Within the study of human cognitive and motor control, appreciable progress has been made in describing the connection between symbolic information integration and complex action learning, opening new lines of research on how verbal instructions influence the neural and cognitive correlates of human behavior (Meiran, Liefooghe, \& De Houwer, 2017; Demanet et al., 2016; Meiran, Pereg, Kessler, Cole, \& Braver, 2015; Everaert, Theeuwes, Liefooghe, \& De Houwer, 2014; Liefooghe, De Houwer, \& Wenke, 2013; Liefooghe, Wenke, \& De Houwer, 2012; Ruge \& Wolfensteller, 2010). In this respect, this study aims to expand upon recent findings on the role of the lateral prefrontal cortex (LPFC) in implementing and memoriz-

${ }^{1}$ Ghent University, ${ }^{2}$ Radboud University Nijmegen either entirely novel or had been trained before the fMRI session. Results replicate higher rMFG-dPMC activation levels during instruction implementation versus memorization and show how this difference is restricted to novel, but not trained, instruction presentations. Pattern similarity analyses at the voxel level further reveal more consistent neural pattern responses in rMFG-dPMC during the implementation of novel versus trained instructions. In fact, this more consistent neural pattern response seemed to be specific to the first instruction presentation and disappeared after the instruction had been applied once. These results further support a role of rMFGdPMC in the implementation of novel task instructions and highlight potentially important differences in studying this region's gross activation levels versus (the consistency of) its response patterns. ing verbal instructions at different levels of information processing and training.

Substantial evidence indicates that successfully implementing instructed mappings between stimuli and motor responses requires processes beyond mere knowledge of these mappings, notably the ability to translate them into motor programs for effective behavior (Brass, Liefooghe, Braem, \& De Houwer, 2017; Everaert et al., 2014; Liefooghe et al., 2012, 2013). This "knowing" versus "doing" divide speaks to the functional segregation between the declarative versus procedural components of working memory, supporting the temporary storage of information for knowledge retrieval and action completion, respectively (Oberauer, 2009; Anderson, 1983). From a clinical standpoint, curtailments in the ability to implement verbally instructed motor rules despite remembering them accurately in patients having sustained major frontal damage following infarct, brain tumors, or head wounds suggest that the procedural component of working memory depends predominantly on the integrity of the frontal lobes (Duncan, 2013; Duncan, Emslie, Williams, Johnson, \& Freer, 1996; Luria, 1966). In 
keeping with this assumption, a number of functional imaging (fMRI) studies have revealed strong linkages between instruction implementation and activity in several areas of LPFC. In one early study, Ruge and Wolfensteller (2010) reported reliable relationships between activity in the right middle frontal gyrus and dorsal premotor cortex (rMFG-dPMC ${ }^{1}$; cf. Figure 2) during instruction encoding and participants' subsequent execution of these instructions. However, that study did not directly compare LPFC involvement in implementing versus memorizing verbal instructions. A more recent study by Demanet et al. (2016) aimed to contrast brain activity during instruction encoding between participants asked to implement stimulus-response mappings on the one hand and participants tasked with memorizing these mappings on the other. Consistent with Ruge and Wolfensteller's (2010) findings, these authors reported a cluster of activity within the rMFG-dPMC in the implementation group only. Together with evidence for right LPFC involvement in praxis-related integration of verbal instructions (Putt, Wijeakumar, Franciscus, \& Spencer, 2017), these findings further suggest a role of this brain sector in translating verbal instructions into motor programs for accurate behavior.

Interestingly, neither of these two studies directly examined the extent to which rMFG-dPMC involvement in novel task instruction implementation can be modulated by participants' level of familiarity with the same instructions. This question appears relevant given evidence for rapid reconfiguration of LPFC activity during repeated execution of motor and cognitive tasks (Ruge \& Wolfensteller, 2010; Raichle et al., 1994). To our knowledge, the only attempt at addressing this question was carried out by Hartstra, Kühn, Verguts, and Brass (2011), whose whole-brain analyses however did not reveal any effect of training on the neural correlates of instruction implementation versus memorization. Instead, they observed left-lateralized LPFC involvement in the processing of novel instructions regardless whether these had to be memorized or implemented. The particular locus of activity reported in this study lay within the ventral portion of the inferior frontal junction (IFJ) - an area that has long received attention for its role in cognitive control and working memory (Brass, Derrfuss, Forstmann, \& von Cramon, 2005; Derrfuss, Brass, \& von Cramon, 2004). This departure from the more systematic findings of the right LPFC involvement in verbal instruction implementation (Putt et al., 2017; Demanet et al., 2016; Ruge \& Wolfensteller, 2010) warrants a more targeted investigation into these regions' neural dynamics during instruction implementation versus memorization at various stages of training.

In this view, we carried out an extensive reanalysis of Hartstra et al.'s (2011) data set using an ROI approach. Our primary goal was to examine whether LPFC might exhibit sensitivity to novel instruction implementation versus memorization following similar neurocognitive patterns as observed in Demanet et al. (2016). In addi- tion, Hartstra et al.'s data set allowed for further investigations into how LPFC activity during instruction implementation versus memorization is modulated by training. In particular, according to computational models of instruction implementation (Ramamoorthy \& Verguts, 2012), the neurocognitive processes involved in early instruction encoding should no longer be necessary whenever the same instructions are repeated, predicting decreases in LPFC involvement from novel to trained instruction implementation in regions presumed to support these processes. Alternatively, evidence exists that parts of LPFC are involved in maintaining arbitrary mappings between stimuli and responses even after training has occurred (Ruge \& Wolfensteller, 2010; Zach, Inbar, Grinvald, Bergmann, \& Vaadia, 2008; Boettinger \& D'Esposito, 2005), predicting sustained or increased involvement of these regions over time. Which areas of LPFC would show one or the other pattern in the particular context of instruction implementation and/or memorization, however, remains unknown.

Importantly, we set out to investigate these questions using both univariate and multivariate (voxel pattern) analyses of fMRI data. In particular, the findings described so far emerged exclusively from univariate fMRI analyses, the conclusions of which are based on macroscopic taskrelated BOLD signal changes averaged over widespread voxel clusters. The explanatory range of such analyses is limited insofar as they remain blind to the contribution of individual voxels within these clusters to explain the neural mechanisms deployed in a given condition. To bridge this gap, advanced multivariate voxel pattern analysis (MVPA) techniques were developed to classify and compare, at a finer level of granularity, the consistency of spatially distributed voxel patterns indicative of these task-specific cognitive mechanisms (Kriegeskorte \& Bandettini, 2007; Kriegeskorte, Goebel, \& Bandettini, 2006; Norman, Polyn, Detre, \& Haxby, 2006) and how they interact with other subcomponents of action planning and execution (e.g., Gilbert, 2011). Importing these methods into research on instruction implementation has allowed researchers to reveal instructed rule identification processes in LPFC areas additional to or overlapping with the regions identified by the studies described above. These include the right ventrolateral LPFC (Reverberi, Görgen, \& Haynes, 2012a, 2012b), left and right IFJ, and anterior portions of the IFS (cf. MuhleKarbe, Duncan, De Baene, Mitchell, \& Brass, 2017; Bode \& Haynes, 2009).

Therefore, besides comparing overall differences in mean activation (i.e., univariate analyses), we also used MVPA to measure the consistency of each region's voxel pattern response to instructions for memorization versus implementation purposes. Specifically, if a given region is involved in instruction implementation by encoding information specific to each new task sequence within a given condition, it should show a different voxel pattern per task sequence. Hence, the consistency in voxel 
pattern response across the different task sequence presentations should be relatively low during instruction implementation and should thus be similar to or even lower than the voxel pattern consistency during instruction memorization. On the other hand, if an ROI subserves an instruction implementation mechanism generalizing over every task sequence (e.g., activating a generic if-then rule) regardless of its individual properties, it should exhibit higher consistency in voxel pattern responses throughout this condition. Within the context of research on the knowing versus doing distinction driving instructed behaviors, this multivariate approach could shed further light upon the complex mechanisms of instruction implementation versus instruction memorization at their different levels of processing and training.

\section{METHODS}

This study features a reanalysis of the data collected in Hartstra et al. (2011). Here we provide a description of the design adapted to the research questions outlined in the Introduction.

\section{Participants}

Participants were 18 right-handed (Oldfield, 1971), healthy individuals (five men, mean age $=21.95$ years, $S D=2.96$ ) without reported history of neurological disorders. Written consent was obtained from all participants before their participation, and the study was approved by Ghent University Hospital ethics review board. A $€ 45$ compensation was provided to participants in return for their participation.

\section{Tasks}

The experimental conditions for this study comprised a stimulus-response condition (SR; cf. Figure 1A) and an object-color condition (OC; cf. Figure 1B). Trials within both SR and OC consisted of task sequences beginning with two task-specific instructions that could or could not be followed by target pictures (see below for detail). Half of these pictures were manmade objects (e.g., CAR), and the other half were animals (e.g., CAT). All pictures were taken from the picture/name database described in Severens, Van Lommel, Ratinckx, and Hartsuiker (2005).

For SR trials, instruction screens provided two stimulusresponse rules linking object or animal names with index finger responses (abbreviated "I") or middle finger responses (abbreviated "M") presented on the left and right side of an equal sign, respectively (e.g., DOG = I; CAT $=\mathrm{M}$ ). Thereafter, a picture could appear, which had or had not been announced as part of the preceding instructions. In the former case, participants had to respond to the target following the instructions given to them by pressing both left and right index or middle fingers simultaneously. These bimanual responses were used to guard against confounding imagery strategies, whereby participants associate objects with particular spatial locations. In the latter case, participants had to refrain from performing any motor responses. Index and middle finger responses for upper and lower instructions were randomized in such a way that one half of index/ middle finger responses were presented as part of the upper instruction line and the other half as part of the lower instruction line. Half of the target pictures were colored differently from the other half. Pink/green, pink/red, and green/red color sets were counterbalanced across participants.
Figure 1. Experimental protocol for the study, illustrating a task sequence in the SR (left, e.g., pressing index/ middle fingers when a jacket/ knife appears) and the OC (right, e.g., verifying whether a banjo/balloon is displayed in red/blue). For both conditions, some instructions would always be followed by one or the other target mentioned on the instruction screen (i.e., applied trials with a response expected), whereas other instructions would be followed by an uninstructed target (i.e., catch trials without expected

responses), and yet others by a blank screen (i.e., instructed trials without expected responses). In addition to these three trial types, control trials were included, in which the instructions could be followed by both instructed targets (as in applied trials) or blanks (as in blank trials). All trials ended with a screen providing feedback information on performance accuracy (see main text for detail). Note that the experiments were conducted in Dutch. 
For OC trials, instruction screens provided participants with stimulus-stimulus rules linking object or animal names and the blue (B) or red (R) colors presented on the left and right side of a "plus" sign, respectively (e.g., Donkey + R; Cat + B). A colored target picture could then appear, which had or had not been announced as part of the preceding instructions. In the former case, participants were asked to establish whether the color of the object matched the object-color mapping rule announced by the instructions by pressing left and right index fingers (abbreviated "I") or middle fingers (abbreviated "M") simultaneously (e.g., true = I; untrue $=\mathrm{M}$ ). The response mappings provided on the target screen were randomized, with one half where index finger responses were presented as part of the upper rule and the other half as part of the lower rule. Furthermore, true or untrue judgment responses were randomly divided over finger pairs so as to prevent associative strategies linking index/middle fingers with particular truth values.

The contrast of interest between SR and OC thus lies in the cognitive processes assumed to take place during the instruction encoding phase. In SR, these processes involve establishing associations between objects and motor responses as early as the instruction phase following an ifthen rule format. OC instructions, by contrast, provide associations between objects and their perceptual features (i.e., color) but do not express any such if-then stimulusresponse rules, which are established only later during the target phase. This difference enabled comparison of verbal instruction encoding with regard to the presence/absence of if-then implementation rules (see Introduction for hypotheses) while closely controlling for the amount and type of information conveyed.

\section{Design and Protocol}

All participants took part in a training session and a scanning session organized on two successive days. The purpose of the training session was to introduce multiple times applied and multiple times instructed SR and OC trials (see below) to be presented again during the scanning session to examine the neural dynamics of repeated instruction implementation or memorization. SR and OC trials were pseudorandomly interspersed throughout the training and scanning sessions with the restrictions that (1) the same task type could not occur five times consecutively and (2) the same instruction screen could not be repeated in two consecutive trials. Both tasks were programmed using E-Prime (Psychology Software Tools). Stimuli in the training session were presented on a 17-in. CRT monitor, and participants' responses were recorded using a standard PC keyboard. Stimuli in the scanning session were presented on an MR-compatible screen visible to participants through a mirror attached to the head coil, and participants' responses were recorded using MR-compatible two-response button boxes placed on their left and right thighs.

\section{Training Session}

Trials in both SR and OC began with a cross-hair displayed in the center of the screen with a random presentation time of $0,50,250$, or $500 \mathrm{msec}$. An instruction screen then appeared for $2000 \mathrm{msec}$, followed by a 250-msec long blank screen. An instructed target, an uninstructed target, or a blank screen (see below for detail) then appeared for a maximum duration of $1500 \mathrm{msec}$ for SR and $2000 \mathrm{msec}$ for OC or until the participant provided a response. Participants were required to respond to instructed targets and to ignore uninstructed targets or blank screens. A 500-msec feedback screen appeared after participants' responses, indicating whether their responses were correct, incorrect, too slow, or with too long a delay between the two finger presses (over $50 \mathrm{msec}$ ).

A total of 160 unique instructions were presented during the session, half of which conveyed implementation rules (SR) and the other half conveyed memorization rules (OC). For each task, 20 of the 80 instructions were followed by a target mentioned in the preceding instruction screen. These instructions were presented 10 times for a total of 200 trials per task. These trials are therefore referred to as multiple times applied trials. For both tasks, half of the target screens displayed one of the instructed targets, and the other half displayed the other target. To counter any strategy formation, whereby participants implement only one of the two instructions and thus prepare only one motor response for each instruction screen, 20 out of the remaining 60 instructions were followed by an uninstructed target. These catch trials were presented one time for a total of 20 trials per condition.

Out of the 40 remaining instructions, 20 of them were never followed by a target. These multiple times instructed trials were presented 10 times, for a total of 200 trials per condition. To guard against strategies whereby participants cease to process instructions that are never followed by a target, the remaining 20 instructions were used as control trials, half of which were followed by a target and the other half by a blank. Each of these 20 instructions was presented four times, for a total of 80 trials per condition.

A training session therefore comprised 500 implementation trials (SR) and 500 memorization trials (OC) for a total of 1000 trials. These were distributed across four blocks of 250 trials each (125 trials per task), with breaks allowed between blocks. Participants were briefed before the training session and were given the opportunity to practice both tasks on 18 trial sequences. None of the items used in these practice blocks reappeared in the experimental blocks. 


\section{Scanning Session}

The scanning session involved carrying out SR and OC while participants' fMRI data were concomitantly being acquired. The overall structure of SR and OC trials was similar to those in the training session, but a pseudologarithmic jitter was introduced between instruction screen offset and target screen onset to separate instructionrelated and target-related brain signal. The jitter was distributed across trials in incremental steps of $800 \mathrm{msec}$, $50 \%$ of which ranged from 200 to $2600 \mathrm{msec}, 30 \%$ from 3200 to $5600 \mathrm{msec}$, and the remaining 20\% from 6200 to $8600 \mathrm{msec}$, for an average jitter time of $3431 \mathrm{msec}$.

One hundred twelve new and unique instructions were used, 56 of which were SR instructions and the other 56 were OC instructions. For both SR and OC, 20 instructions were always followed by an instructed target and were presented twice over the scanning session for a total of 40 trials per condition. For half of these newly applied trials, one of the instructed targets was presented on the target screen, and for the other half, the other target. To guard against strategies whereby participants processed/prepared only one instruction/motor response, 6 of the remaining 36 instructions were used as catch trials, in which instruction screens were followed by uninstructed targets.

Of the remaining 30 instructions, 20 of them were never followed by a target. These newly instructed trials were presented twice for a total of 40 trials per condition. To ensure that participants effectively processed these instructions, the 10 remaining instructions were used four times in control trials, half of which displayed a target and the other half a blank, again totaling to 40 trials per task. In summary, 252 trials employed new and unique instructions that were not used in the training session.

In addition to these new trials, the 20 multiple times applied and 20 multiple times instructed trials per task of the training session were also used in the scanning session. Instructions in all of these trials were followed by an instructed target and presented twice for a total of 160 trained instruction trials ( 80 per task).

The scanning session therefore comprised 412 trials distributed across four experimental blocks. Participants were briefed about the SR and OC conditions and were given the opportunity to practice both conditions for 18 trials. None of the stimuli used in the practice phase were used in the experimental phase. Overall, the scanning sessions lasted about $50 \mathrm{~min}$.

\section{Behavioral Data Analysis}

Besides checking overall performance accuracy, behavioral data analyses were conducted primarily to examine the possible effects of participants' familiarity with instructions on their response latencies and performance accuracy. To this end, response latencies (in milliseconds) and percent accuracy scores for the first and second iterations of the newly applied and multiple times applied trials were entered into repeated-measures ANOVAs including Training (newly applied vs. multiple times applied, two levels) and Iteration (first vs. second iteration, two levels) as within-subject factors. This was done separately for SR and OC.

\section{fMRI Acquisition and Pre-processing}

The scanning sessions were run on a 3T Siemens Trio scanner using an eight-channel radiofrequency head coil. Participants lay supine in the scanner while 176 high-resolution structural scans were first acquired through a T1-weighted 3-D MPRAGE sequence (repetition time $=2.53 \mathrm{sec}$, echo time $=2.58 \mathrm{sec}$, image matrix $=$ $256 \times 256$, field of view $=220 \mathrm{~mm}$, flip angle $=7^{\circ}$, slice thickness $=0.90 \mathrm{~mm}$, voxel size $=0.9 \times 0.86 \times 0.86 \mathrm{~mm}$ resized to $1 \times 1 \times 1 \mathrm{~mm}$ ). Whole-brain functional volumes were then acquired while participants performed the tasks using a T2*-weighted EPI sequence (repetition time $=2 \mathrm{sec}$, echo time $=35 \mathrm{msec}$, image matrix $=64 \times 64$, field of view $=224 \mathrm{~mm}$, flip angle $=80^{\circ}$, slice thickness $=3.0 \mathrm{~mm}$, distance factor $=17 \%$, voxel size $=3.5 \times 3.5 \times 3.5 \mathrm{~mm}$, 30 axial slices). Given the self-paced initiation of trials, a varying number of volumes were acquired per block.

MRI data preprocessing was performed using SPM8 (http://www.fil.ion.ucl.ac.uk/spm/software/spm8/). The first two scans of each EPI series were removed to control for T1 relaxation effects. For each participant, a mean image for all volumes was produced, and individual volumes were spatially realigned using rigid body transformation. The volumes were then slice time-corrected using the first slice as reference. Each participant's structural image was then coregistered with their mean functional image and normalized to the Montreal Neurological Institute T1 template (Montreal, Quebec, Canada). The four separate runs were concatenated into a single session. Both analysis types were also performed on temporally smoothed data using a $128-\mathrm{Hz}$ high-pass filter. Images were resampled into $3.5 \mathrm{~mm}^{3}$ voxels.

An important difference between the preprocessing steps preceding univariate and multivariate fMRI analyses relates to the use of FWHM spatial smoothing applied to voxel assemblies. Although such procedure is typically used to increase signal-to-noise ratio in whole-brain univariate analyses, it is generally avoided in multivariate analyses as it defeats their purpose of accounting for the contribution of individual voxels as part of their results (Kriegeskorte \& Bandettini, 2007; Kriegeskorte et al., 2006; Norman et al., 2006). In effect, in the present case, spatial smoothing proved unnecessary for either the univariate or the multivariate analyses, because both were conducted on MR signal obtained from predefined ROIs (see below). Even for univariate analyses, spatial smoothing applied over ROIs is not only redundant but also likely to mix signal from voxels within these ROIs with signal from outside neighboring voxels. For these 
reasons, spatial smoothing was not applied before either the univariate or the multivariate analyses.

\section{fMRI Analyses}

Regions of Interest

The specific ROIs selected for analysis are featured in Figure 2. Of primary interest to this study is the activation cluster reported in Demanet et al. (2016), which spanned from the middle aspects of the right frontal gyrus back to the dorsal premotor cortex (Figure 2, violet). Although this cluster was originally labeled by Demanet et al. (2016) the IFS because of its observed peak coordinates (MNI, $x=39, y=20, z=31$ ), its anatomical overlap and resemblance with the cluster reported earlier by Ruge and Wolfensteller (2010) as the rMFG-dPMC motivated us to choose the latter label for consistency. The ROI used for this cluster is the original cluster of activity observed by Demanet et al. (2016). Additional ROIs examined here included the left and right IFJ (cf. Figure 2, turquoise) and the left and right IFS (cf. Figure 2, blue), both of which were examined in the context of MuhleKarbe et al.'s (2017) study on instruction implementation versus memorization and constructed as $10-\mathrm{mm}$ radius spheres centered around the center-of-gravity coordinates reported in Muhle-Karbe et al. (2017): IFJ left: $x=-40, y=4, z=30$; IFJ right: $x=44, y=10, z=34$; left and right IFS were $x= \pm 38, y=39, z=23$. Finally, we also included the right ventrolateral pFC (VLPFC; yellow), reported as part of the studies of Reverberi et al. (2012a, 2012b) on rule identification processes and provided to us courtesy of Dr. Carlo Reverberi (University of Milano-Bicocca, Milan).

It is worth mentioning at this point that these ROIs were observed and/or investigated as part of studies using very different instruction formats, including exclusively verbal instructions (Demanet et al., 2016), mixtures of abstract patterns and verbal instructions (Ruge \& Wolfensteller, 2010), mixtures of real-world pictures and verbal instructions (Muhle-Karbe et al., 2017), or abstract patterns exclusively (Reverberi et al., 2012a, 2012b). It is therefore quite possible that the findings reported across these studies may differ as a result of differences in these instructions' modality. Including these ROIs as part of this study allowed us to additionally investigate which parts of LPFC may preferentially respond to instructions conveyed in an exclusively verbal format.

\section{General Linear Model}

A first general linear model (GLM) of the data was obtained using the GLM tool implemented in SPM8. Because this study focused on the instruction phase in both SR and OC trials, the main focus was on the onset of the instruction screens, on which participants' canonical hemodynamic response function was modeled along with its first derivative. The primary analyses were designed to investigate the neural dynamics of each ROI associated with instruction implementation versus memorization at their different levels of information processing and training. This was done by comparing brain responses to instructions pertaining to trials carried out during the scanning session only ("novel" instructions) and instructions pertaining to trials performed in the training session and repeated in the scanning session ("trained" instructions). In the primary GLM analysis, novel instructions were obtained by including within a single vector the first instruction screens of the newly applied trials and the first instruction screens of the newly instructed trials (see also Hartstra et al., 2011, for a similar approach). The sole difference between these trial types is that, in the latter case, instructions were never followed by a target; therefore, both trial types were similar with regard to their instruction phase. This was done separately for OC and SR. The second instruction screens of the newly applied and newly instructed trials were included as regressors of noninterest. Instruction screens for trained instructions included the first and the second

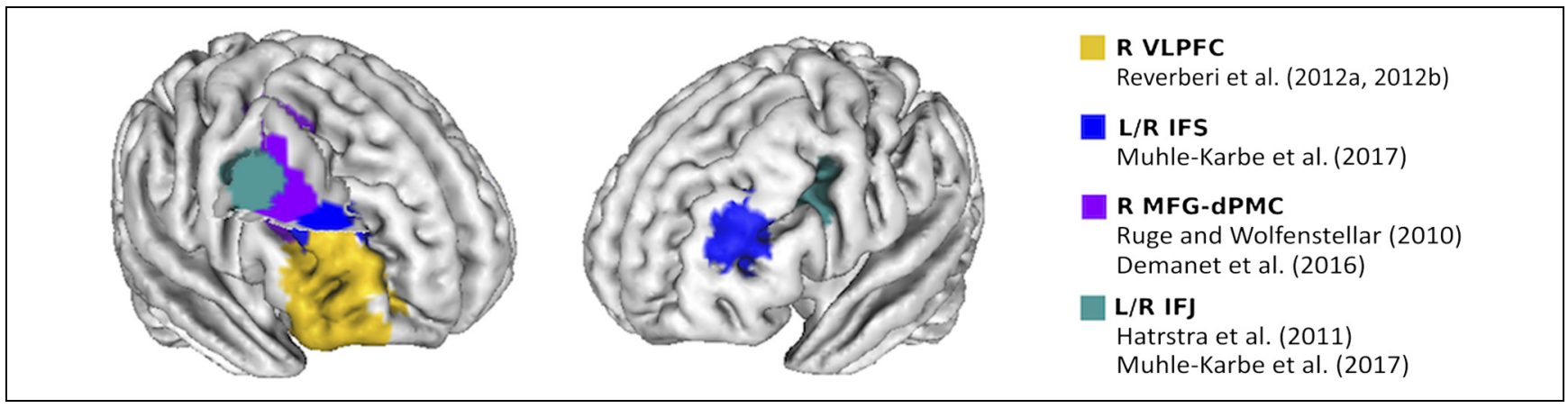

Figure 2. ROIs selected for analysis and overlaid on the standard MNI template. The results presented here focus on the rMFG-dPMC, the right VLPFC (R VLPFC), the left and right IFJ (L/R IFJ), and the left and right IFS (L/R IFS). References given next to each ROI correspond to studies in which this ROI displayed sensitivity to instruction onset. The ROI for the rMFG-dPMC is the original cluster of activity reported in Demanet et al. (2016). The ROI for the right VLPFC was build based on the cluster of activity reported in Reverberi et al. (2012a, 2012b) and obtained through the courtesy of Carlo Reverberi (University of Milano-Bicocca, Milan), and ROIs for left and right IFJ and left and right IFS were built as 10-mm radius spheres centered around the center-of-mass coordinates, as also used in Muhle-Karbe et al. (2017). 


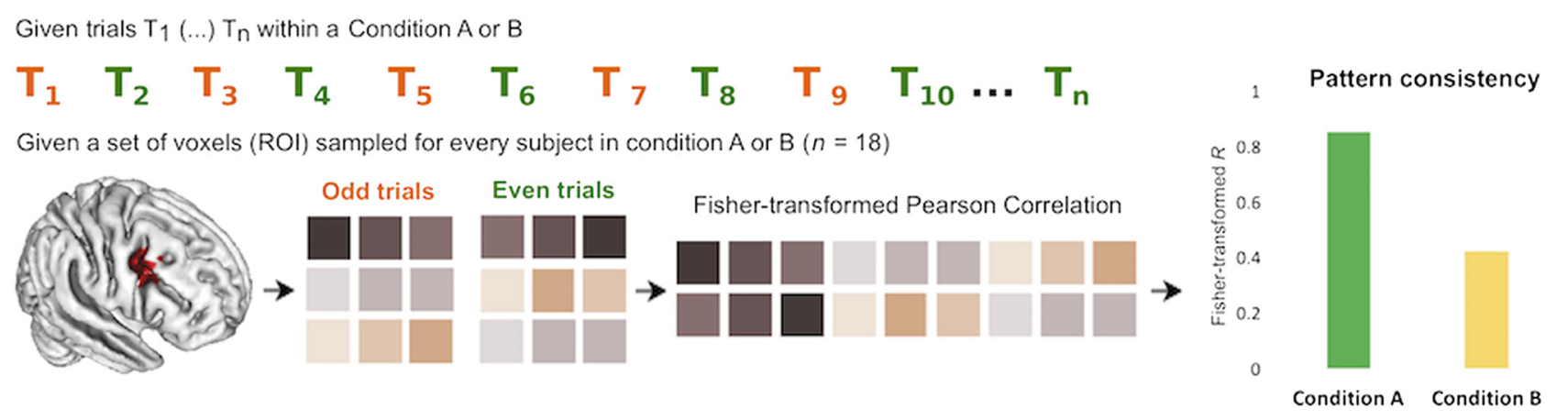

Figure 3. Procedure used in voxel pattern analyses of the present data. Analyses were performed following a split-half testing method of internal consistency, whereby GLM trials within each condition of interest were distributed into separate vectors depending on being in an odd or even position. Next, we performed Fisher-transformed Pearson correlations between the voxel patterns for odd- and even-trial vectors for the same condition in each participant, as a measure of voxel pattern consistency. The resulting condition-specific correlations were then averaged and entered into repeated-measures ANOVAs for comparisons.

instruction screens of the multiple times applied trials. Once again, this was done separately for SR and OC. Regressors of noninterest comprised brain activation during the first and second instruction screens of the multiple times instructed SR and OC trials, brain activation related to target screen presentation, catch and control trial instructions. Finally, six regressors were also included to model residual motion artifacts.

\section{Univariate Analyses}

Univariate analyses of the data were based on task-related activation of each ROI averaged within each condition, that is, novel SR, trained SR, novel OC, and trained OC. This was done separately for each participant. The average activation patterns were then entered into repeatedmeasures ANOVA for analysis (see Univariate Analyses in the Results section for details on the factors included in the ANOVAs).

\section{Multivariate Voxel Pattern Analyses}

In line with previous research from our group (Braem et al., 2017), MVPA was performed to assess and compare pattern consistency per condition - that is, the degree to which voxel patterns between trials of the same conditions more or less correlated with one another (cf. Figure 3). This approach is reminiscent of the notion of internal consistency as defined within classical test theory (McDonald, 1999), for which the correlation between items belonging to the same tests reflects the degree to which these tests accurately measure a given attribute or psychometric construct (e.g., fluid intelligence, creativity, visuospatial reasoning). Similarly, the degree to which data points within the same condition (in this case SR or OC) or subcondition (novel or trained) correlate with one another should signal the presence of mechanisms specific to this (sub)condition but shared across the different task exemplars. In the present case, these mecha- nisms could include (a) recognition of instructions as novel or trained, (b) encoding of stimulus-response rules (associating a given perceptual stimulus with a motor response, as in SR) or stimulus-stimulus rules (associating the identity of the stimulus with a perceptual attribute such as color, as in OC), or (c) a combination of both (i.e., novel vs. trained stimulus-response rules in novel SR vs. trained SR trials or novel vs. trained stimulus-stimulus rules in novel vs. trained OC trials). Because a different set of instructions was presented for each trial (i.e., task sequence), these correlations were within condition but across task sequences. Alternatively, condition-specific involvement of a brain region could also result in indifferent (or even lower) internal consistency measures. Specifically, if a given region also processed or retained task-specific information during one type of instruction presentation specifically (e.g., the processing of novel stimulus-response rules), one would expect more dissimilarity in voxel pattern responses to the different task exemplars and thus not a higher internal consistency. To this end, we tested whether (1) regions showed internal consistency measures during the implementation of novel stimulus-response rules that differed from novel stimulus-stimulus rules or trained stimulus-response rules and (2) used the direction of this difference to infer whether this region processed task-specific information or recruited a more abstract process specific to novel task implementation instead.

Following the split-half method of internal consistency estimation (McDonald, 1999; Cronbach, 1951), the instructions modeled in the novel and trained instruction vectors for SR and OC in the GLM described in the General Linear Model section were distributed across two separate regressors depending on being odd or even data points within the vector. Representational similarity analyses were then performed on participants' unsmoothed parameter estimates using custom made scripts (developed by Kenneth S. L. Yuen at Neuroimaging Center 
Mainz, Johannes Gutenberg University Medical Center, Germany). For each ROI examined, the processing steps for these analyses included (1) extraction of individual participants' parameter estimates for odd and even trials within each condition, (2) correlation of individual participants' parameter estimates between odd and even trials within each condition, and (3) Fisher transformation of individual participants' correlation coefficients allowing for statistical comparisons. The data were then entered into repeated-measures ANOVAs (see Results for more detail on the factors included in the ANOVAs).

\section{Follow-up MVPA}

In a second set of multivariate analyses, we examined the impact of having previously applied or merely received (but not applied) an instruction within the context of the scanning session only. To this end, we constructed a second GLM in which separate vectors were created for the first and second presentation screens of the newly applied and newly instructed trials separately. This was done separately for SR and OC. The main differences between these trials and those used for the primary analyses are (1) that their repetition is restricted to two iterations during the scanning session (thus permitting comparison of internal consistency between the first and the second iteration of the same task sequence) and (2) that instructions during the newly instructed trials are never actually executed in the target phase (thus permitting comparison of internal consistency between brain signal associated with effectively executed task rules and rules that were instructed before but never executed). Similar to the primary analyses, trials within each of these separate vectors were distributed between separate regressors, depending on their position as odd or even data points within the vector. Once again, the goal of this odd/even distribution was to be able to compare internal consistency between odd and even trials of the first and second presentation screens. Regressors of noninterest modeled brain activation related to target screen presentation, catch and control trial instructions, and six additional regressors were also included to model residual movement-related artifacts.

\section{RESULTS}

\section{Behavioral Data}

More detailed behavioral analyses of the same data set are described in Hartstra et al. (2011). Given the focus of this study, we were interested here mainly in the extent to which previous applications of instructions presented during the training and scanning sessions (multiple times applied trials) or during the scanning session only (newly applied trials) impacted participants' performance during scanning. We predicted in particular that participants should learn from previous task applica- tions, which should be reflected in progressive decreases in response latency and, conversely, increases in performance accuracy. To this end, we entered RTs for the first and second iterations of the multiple times applied trials and newly applied trials in a repeated-measures ANOVA including Training (newly vs. multiple times applied trials, two levels) and Iteration (first vs. second trial) as within-subject factors. This was done for SR and OC separately. As predicted, RTs during the scanning session decreased as participants' level of training with instructions increased (cf. Figure 4, top left and right graphs). This effect was reliable both between newly applied and multiple times applied trials of both SR and OC (main effect of Training in SR: $F(1,17)=129.00, p<.001, \eta_{\mathrm{p}}^{2}=.884$ and OC: $\left.F(1,17)=17.093, p=.001, \eta_{\mathrm{p}}^{2}=.501\right)$. The same effect was also observed between the first and second iteration of both newly applied and multiple times applied SR trials (main effect of Iteration: $F(1$, $\left.17)=12.864, p=.002, \eta_{\mathrm{p}}^{2}=.431\right)$ but not for OC trials $(F(1,17)=2.339, p=.145)$. No reliable Training $\times$ Iteration interaction was significant for either SR or OC (all Fs $<1)$.

Also in line with predictions, percent accuracy scores increased between newly applied and multiple times applied trials (cf. Figure 4, bottom left and right graphs), as indicated by a main effect of Training in both SR $(F(1$, $\left.17)=10.442, p=.005, \eta_{\mathrm{p}}^{2}=.381\right)$ and OC $(F(1,17)=$ 21.235, $\left.p<.001, \eta_{\mathrm{p}}^{2}=.555\right)$. There was a trend toward greater accuracy from the first to the second iteration of SR trials (main effect of Iteration: $F(1,17)=3.457, p=$ $.08)$, which was not apparent for OC (Iteration: $F(1$, $17)=2.565, p=.127)$. No reliable Training $\times$ Iteration interaction was obtained for either SR or OC (all $F$ s $<1)$. Accuracy remained safely above chance levels for all participants (over 65\%) as well as at the group level (over 80\%), confirming that they understood the tasks and had sustained attention throughout the scanning session.

\section{fMRI Analyses}

\section{Univariate Analyses}

Results of the univariate analyses are displayed in Figure 5. As outlined above, the first goal of this study was to replicate Demanet et al.'s (2016) initial observation that rMFG-dPMC supports instruction implementation processes additional to simple instruction memorization (SR vs. OC) in the absence of training. We investigated this question with a first repeatedmeasures ANOVA using ROI (six levels) and Condition (novel SR vs. novel OC, two levels) as factors of interest. Analyses revealed a reliable ROI $\times$ Condition interaction $\left(F(3.334,56.685)=9.23, p<.001, \eta_{\mathrm{p}}^{2}=\right.$ .352). We followed up on this interaction with paired $t$ tests comparing BOLD signal amplitude between novel SR and novel OC conditions in all ROIs. Results 
revealed significantly larger BOLD signal in novel SR compared with novel OC in right IFJ $\left(t_{17}=4.064\right.$, $p=.006)$ and rMFG-dPCC $\left(t_{17}=3.409, p<.02\right.$, all other $p s$ not significant).

In addition, we examined whether this putative implementation-specific function of rMFG-dPMC pertains to the implementation of novel task instructions or persists across novel and trained task sets. We addressed this question with a second repeated-measures ANOVA, including ROI (six levels), Condition (SR vs. OC, two levels), and Training (novel vs. trained) as factors of interest. Results revealed a reliable ROI $\times$ Condition interaction $\left(F(2.829,48.096)=5.733, p=.002, \eta_{\mathrm{p}}^{2}=.252\right)$, a reliable Condition $\times$ Training interaction $(F(1,17)=$ $\left.6.437, p=.021, \eta_{\mathrm{p}}^{2}=.275\right)$, and a reliable ROI $\times$ Condition $\times$ Training interaction $(F(3.328,56.580)=4.949, p=$ $\left..003, \eta_{\mathrm{p}}^{2}=.225\right)$. When following up on this by studying the Condition $\times$ Training interaction for each ROI separately, this interaction was reliable in the left IFJ ( $F(1$, $\left.17)=7.506, p=.014, \eta_{\mathrm{p}}^{2}=.306\right)$, the right IFJ $(F(1$, 17) $\left.=15.509, p=.001, \eta_{\mathrm{p}}^{2}=.477\right)$, as well as the rMFG-dPMC $\left(F(1,17)=15.652, p=.001, \eta_{\mathrm{p}}^{2}=.479\right)$, but not in the other regions (all $p s>.37$ ). In the right IFJ and rMFG-dPMC, this interaction confirmed that the implementation-specific function of these regions was specific to novel task instructions. Specifically, the interaction was driven by a reliably larger BOLD signal change in novel SR versus novel OC (see above), but this difference was absent on the trained SR versus OC trials $(p s \geq .06)$. In the left IFJ, the interaction was primarily driven by a significantly larger BOLD signal in novel compared with trained SR $\left(t_{17}=2.742, p=.01\right)$.

It is worth remarking on the unexpectedly high BOLD signal amplitude observed for trained SR and OC trials in right IFJ and rMFG-dPMC. Because this finding was not part of our main research question, it will not be investigated further here. However, we will formulate some speculative avenues of interpretation regarding its functional significance in the Discussion section.

\section{MVPA}

The next set of analyses reported in this study aimed to investigate, from a multivariate angle, whether these ROIs also show differences in the consistency of their voxel pattern responses. Such differences would not only further support their role in the implementation of novel stimulus-response rules (SR trials) but also give further insight as to whether their role is one that also codes for specific task information (i.e., similar or lower voxel pattern consistency) or recruits a more abstract process
Figure 4. Evolution of RTs and performance accuracy as a function of the first and second iteration (It1 and It2) of newly applied trials (NA) and multiple times applied trials (MTA) of the SR $(\mathrm{A} / \mathrm{C})$ and the OC $(\mathrm{B} / \mathrm{D})$ during the scanning session.

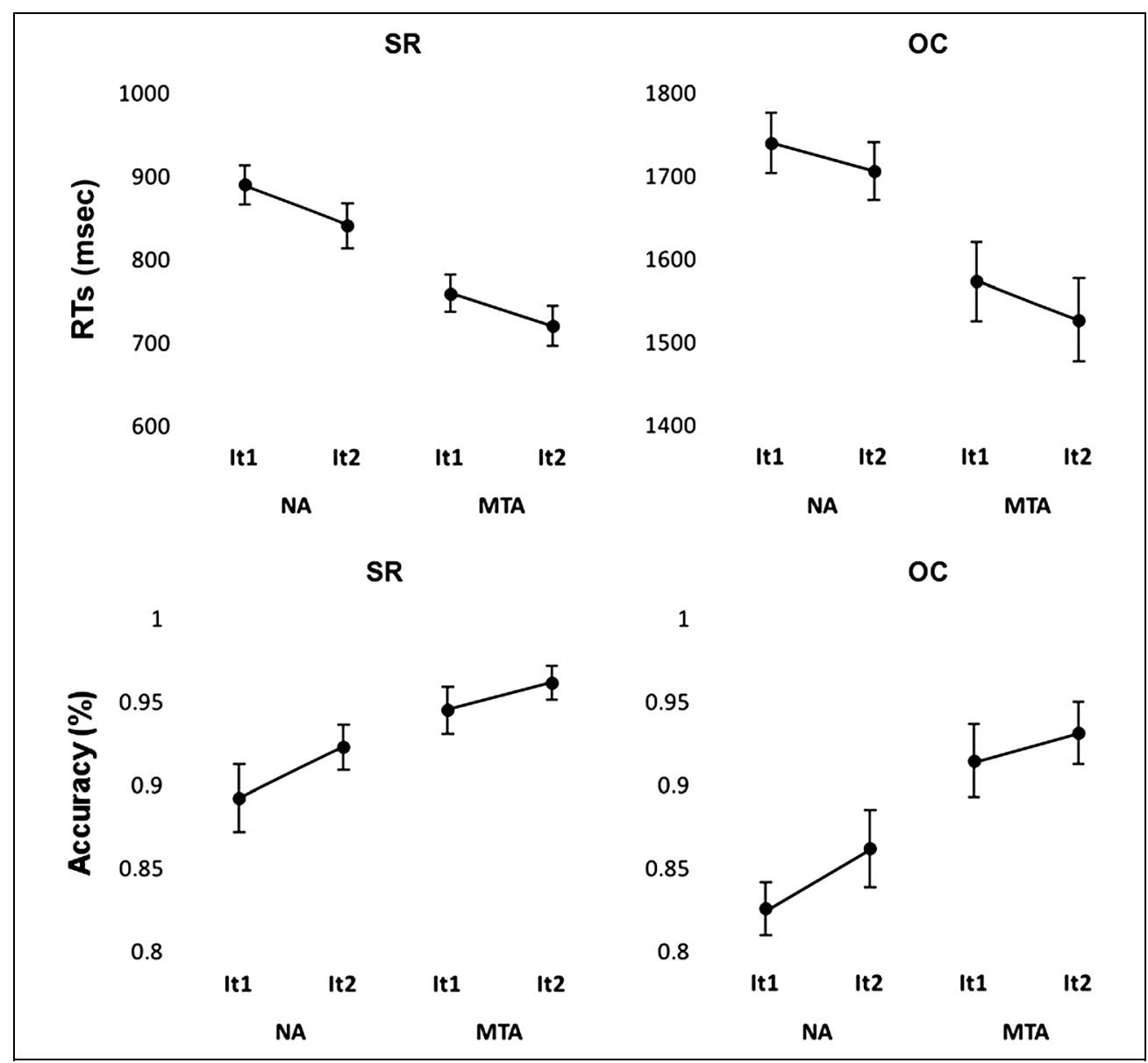




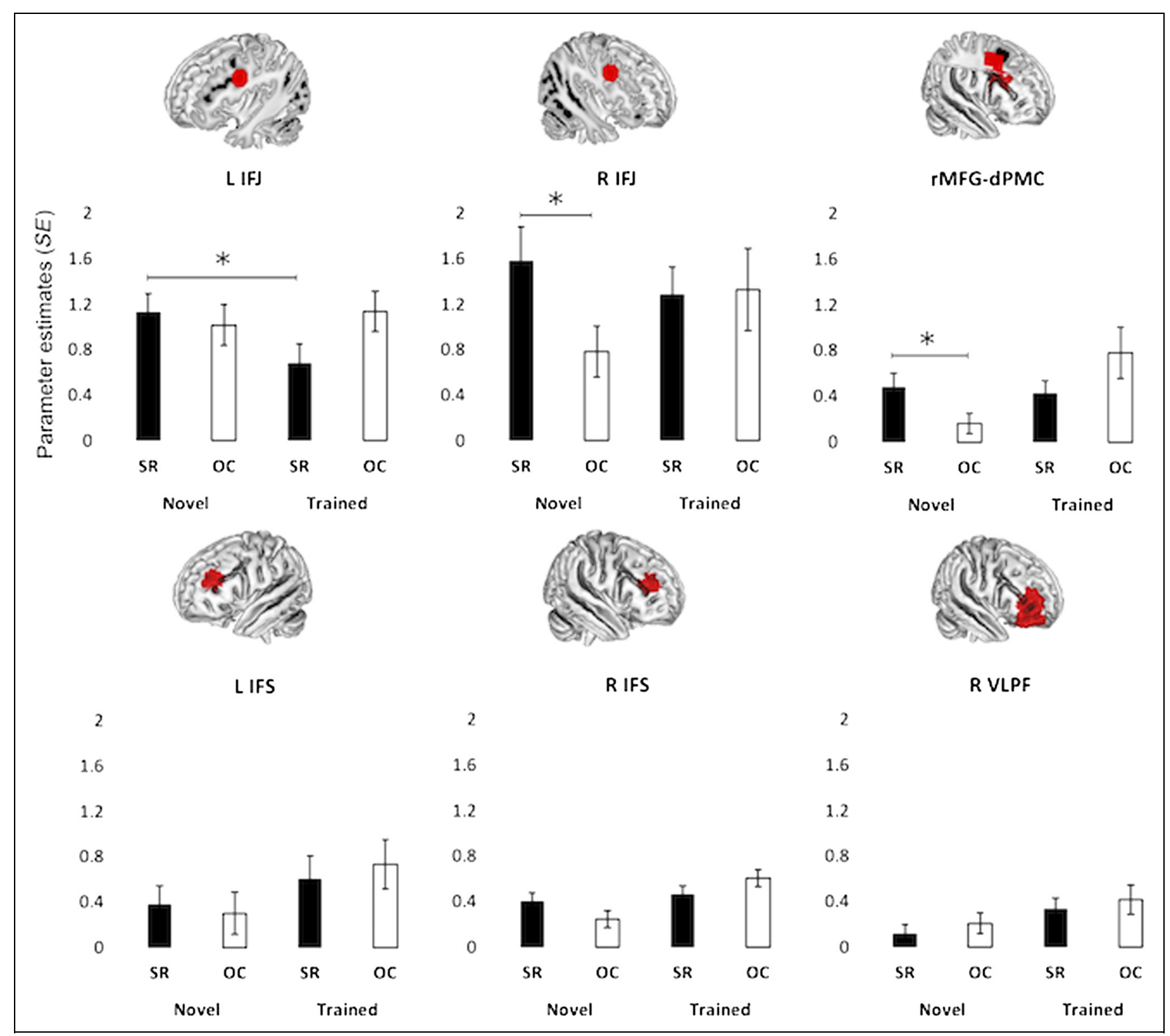

Figure 5. Mean parameter estimates (with standard errors of mean across participants) from the univariate analyses comparing novel and trained trials of the SR and the OC in the left/right IFJ (R/L IFJ), the left/right IFS (L/R IFS), the rMFG-dPMC, and the right VLPFC (R VLPFC). * $p<.05$.

that generalizes across tasks instead (i.e., higher voxel pattern consistency).

Results of the multivariate analyses are displayed in Figure 6. Following the same approach as in the univariate analyses, we first investigated the extent to which reliable differences could be observed between novel SR and novel OC instructions also at the multivariate level. We first performed a repeated-measures ANOVA on the Fisher-transformed Pearson's $R$ correlations between odd and even trials of novel SR and novel OC, which revealed a reliable ROI $\times$ Condition interaction $(F(3.367,57.235)=$ $4.581, p=.005, \eta_{\mathrm{p}}^{2}=.212$ ). Follow-up $t$ tests indicated a trend toward greater internal consistency in SR compared with OC in right IFJ $\left(t_{17}=2.041, p=.057\right)$ and rMFGdPMC $\left(t_{17}=1.769, p=.095\right.$, other $p$ s not significant $)$. This higher similarity in voxel patterns between the odd and even trials of the novel SR condition suggests that these regions possibly recruit an abstract process that helps implementing stimulus-response rules (but not so much stimulus-stimulus rules), irrespective of the exact task information.

To examine the effect of training on the differences between SR and OC in these regions' voxel pattern consistency, we ran another repeated-measures ANOVA including Training (novel vs. trained, two levels) as additional within-subject factor. Results revealed a reliable ROI $\times$ Condition $\times$ Training interaction $(F(1,17)=$ $\left.3.968, p=.01, \eta_{\mathrm{p}}^{2}=.189\right)$. Interestingly, the only region showing a significant Condition $\times$ Training interaction was the rMFG-dPMC $\left(F(1,17)=5.06, p<.04, \eta_{\mathrm{p}}^{2}=\right.$ $.229)$, whereas all other regions showed no such interaction (all $p s>.09$ ). Similar to the univariate analyses, 
follow-up $t$ tests for rMFG-dPMC again showed how there was a significant difference between SR and OC when they were novel (see above), but not when this comparison was done on trained task sequences $\left(t_{17}=1.061, p>.3\right)$. Moreover, these multivariate analyses showed greater pattern consistency in novel SR compared with trained SR $\left(t_{17}=\right.$ 2.893, $p=.01)$. Importantly, this result indicates that odd- and even-trial vectors within the novel SR condition display more similarity in their voxel patterns than oddand even-trial vectors within the trained SR condition and suggest that the rMFG-dPMC hosts an abstract process that is most responsive to the implementation of novel stimulus-response rules (but not necessarily retains task-specific information). Arguably, this effect could be driven by the higher mean activation in novel SR than trained SR trials (albeit not significantly so; see Figure 5), because a higher BOLD signal amplitude is likely to increase pattern reliability (the proportion of the response accounted for by signal relative to noise is higher). To rule out this possibility, we ran a repeated-measures ANCOVA contrasting mean Fisher-transformed Pearson correlation coefficients between novel and trained SR while including the difference in BOLD signal amplitude between novel SR and trained SR as confounding variable. Results showed that controlling for potential differences in BOLD signal amplitude did not significantly affect the difference in voxel pattern consistency between novel and trained $\mathrm{SR}\left(F(1,17)=7.486, p=.015, \eta_{\mathrm{p}}^{2}=.319\right)$.



Figure 6. Mean Fisher-transformed Pearson coefficients (with standard errors of mean across participants) comparing within-task voxel pattern consistency (even vs. odd trials) between the implementation task (SR) and the memorization task (OC) in the left/right IFJ (L/R IFJ), the left/right IFS (L/R IFS), the rMFG-dPMC (red ROI), and the right VLPFC (R VLPFC). Correlation coefficients (with standard errors across participants) were Fisher-transformed before statistical analyses. $0.1<p>.05, * p<.05$. Error bars reflect standard errors of mean across participants. 
Figure 7. Results from the follow-up MVPA comparing the first and second iteration of newly applied (APL 1 vs. 2) and newly instructed (INST 1 vs. 2) trials of the SR and OC task in rMFG-dPMC. $* p<.05, * * p<$ .01 . Error bars indicate standard errors of mean across participants.

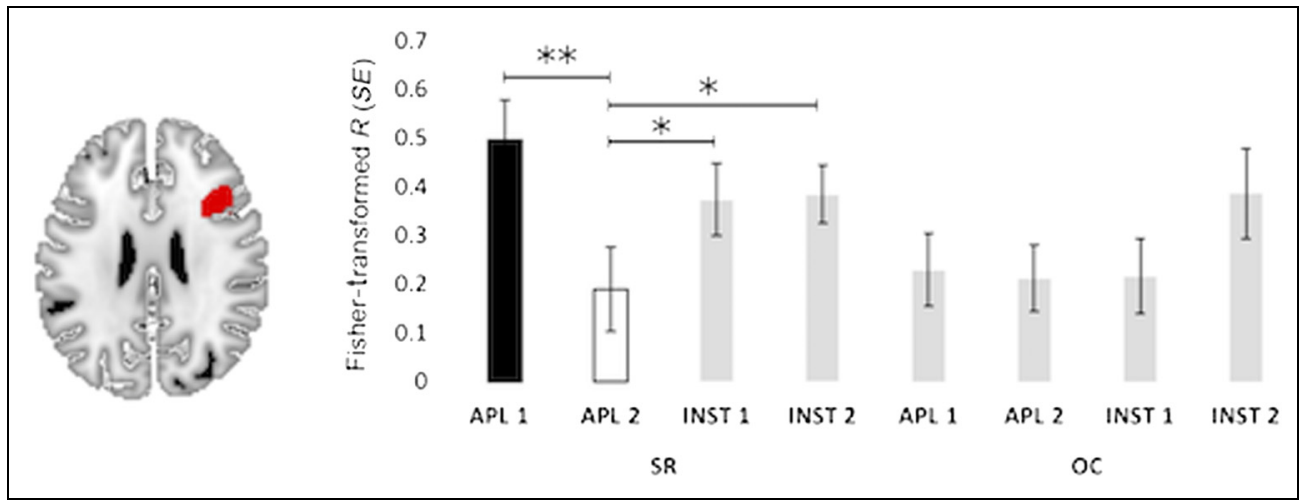

\section{Follow-up MVPA in rMFG-dPMC}

Interestingly, the conception of rMFG-dPMC processes as being deployed in encoding task rule representations exclusively in the context of novel instructions raises questions about the amount of training necessary for these processes to become automatized. In this respect, the primary analyses in this study focused on distinguishing brain responses to novel instructions from responses associated with instructions repeated a substantial amount of times in the training session (approximately 10 times for each trained trial). However, previous evidence for rapid reconfiguration of brain activity over the first few repetitions of initially novel instructions (Ruge \& Wolfensteller, 2010) suggests that the putative training-related modulation of rMFG-dPMC processes in instruction implementation may be detected already from the first to the second iteration of entirely novel instructions, predicting reliable decreases in voxel pattern consistency already from the first to the second presentation of a new trial. Second, the notion that differences in internal consistency in right rMFG-dPMC activity reflects training-related modulations of task rule encoding processes for prospective action entails that these modulations are contingent upon effectively executing instructions in the target phase. From this follows the prediction that internal consistency should decrease only in the context of applied rather than instructed trials.

To this end, we ran a separate GLM (as described in the Methods section), which involved (odd and even trial) regressors coding for first instructed and applied task rules (APL1), second instructed and applied task rules (APL2), first merely instructed task rules (INST1), and second merely instructed task rules (INST2; cf. Figure 7). Insofar as rMFG-dPMC responds most consistently to instructions that have not yet been applied, its response pattern consistency should decrease as early as on its second presentation. We tested this hypothesis by entering Fishertransformed Pearson's $R$ coefficients between the odd and even trials of APL1, APL2, INST1, and INST2 instructions in SR into repeated-measures ANOVAs including the factors Application (instructed and applied vs. merely instructed, two levels) and Presentation (first vs. second presentation, two levels) as within-subject factors. The ANOVA yielded a reliable main effect of Presentation $(F(1,17)=5.305, p<$ $\left..04, \eta_{\mathrm{p}}^{2}=.238\right)$, as well as a highly reliable Application $\times$ Presentation interaction $\left(F(1,17)=20.479, p<.001, \eta_{\mathrm{p}}^{2}=\right.$ .546). This interaction was driven by a lower level of consistency for APL2 compared with APL1 $\left(t_{17}=-3.861, p=\right.$ .001 ), whereas no reliable difference was observed between the first and the second presentation of the merely instructed trials $\left(t_{17}=-0.187, p>.8\right)$, underpinning the notion that response pattern consistency in rMFG-dPMC is operationalized by effectively applying instructions. Although visual inspection of Figure 7 suggests lower internal consistency for INST1 and INST2 relative to APL1, paired comparisons did not bear meaningful differences between these conditions (all $p s>.07$ ), once again bolstering the claim that this effect in rMFG-dPMC is specific to instruction implementation. The same repeated-measures ANOVA performed on OC trials yielded no reliable effects or interactions (all $p s>.1$ ).

\section{DISCUSSION}

Research on the shaping role of verbal instructions on behavior has witnessed a surge of new findings underpinning the central role of LPFC in the capacity to quickly transform instructed stimulus-response mappings into effective motor programs for prospective action. This capacity has previously been associated with the procedural component of working memory in charge of action completion beyond mere knowledge retrieval (i.e., the declarative component of working memory; cf. Oberauer, 2009) and appears to be heavily dependent on the integrity of LPFC (Duncan, Hazel, Williams, Johnson, \& Freer, 1996; Duncan et al., 2008). At the same time, the relative contribution of specific LPFC areas in verbal instruction implementation has remained underspecified. As an attempt to replicate and expand upon recent work suggesting a possible role of rMFG-dPMC in verbal instruction implementation versus memorization (Demanet et al., 2016), this study sought to (1) understand whether this and neighboring LPFC areas would exhibit similar sensitivity to to-beimplemented (SR) versus to-be-memorized instructions 
(OC), (2) further document the neural dynamics of these areas in instruction implementation versus memorization as a function of training, and (3) approach these questions from the perspective of univariate versus multivariate (i.e., MVPA) analyses of functional imaging data, the latter approach allowing for deeper understanding of the exact role of these regions in implementing novel task instructions (e.g., retaining task-specific information or a more general process of instruction implementation at different levels of processing and training).

Replicating and extending Demanet et al.'s (2016) findings, univariate analyses revealed stronger activity in rMFG-dPMC and right IFJ in response to novel SR compared with novel OC instructions, supporting these regions' sensitivity to novel instructions conveying explicit stimulus-response mappings as opposed to stimulusstimulus mappings. Interestingly, the same regions also exhibited a relatively high level of activation in response to novel and trained SR instructions. This observation is in line with previous research highlighting the importance of LPFC and neighboring premotor regions in supporting arbitrary sensorimotor mappings even after training has occurred (Ruge \& Wolfensteller, 2010; Zach et al., 2008; Deiber et al., 1997) and suggests that rMFGdPMC and IFJ may play similar functions in the particular context of verbal instruction implementation. The BOLD signal increase from novel OC to trained OC was particularly unexpected and may be interpreted after the fact as the result of having performed motor responses during the truth-value judgments of instructed object-color mappings. In contrast to novel or trained SR instructions, these motor responses are not explicitly mentioned as part of the instruction phase and are established only subsequently as part of the target phase. In this view, repeated OC instructions would proactively trigger motor responses that were previously established and performed during target phases. The main difference between this study's SR and OC task sequences when it comes to stimulus-response mappings would thus lie in the exact point within these sequences where motor responses are established (i.e., instruction phase in SR vs. target phase in OC). Some evidence from previous imaging research on working memory seems to support this proposition (Pochon et al., 2001; Rowe, Toni, Josephs, Frackowiak, \& Passingham, 2000), although more work is needed to understand the effect of timing of motor response selection on the neural correlates of instruction implementation versus memorization.

Although univariate results indicate a possible role of rMFG-dPMC and IFJ in maintaining new or previously learned sensorimotor mappings, MVPA can provide more sensitive insights into the cognitive processes involved in assimilating these sensorimotor mappings on the basis of verbal instructions. In this regard, our MVPA results revealed more consistent voxel patterns in novel versus trained SR confined to rMFG-dPMC, suggesting that this region harbors information processing mechanisms spe- cific to novel instruction implementation regardless of instruction identity. These mechanisms are unlikely to pertain to stimulus classification, as these comprised similar object classes (manmade objects or animals) for both SR and OC trials. Rather, the main factor distinguishing SR from OC instructions is the relation between stimuli and motor responses. The fact that pattern consistency decreases as early as the second iteration of previously applied instructions and persists as long as instructions have not been applied (cf. follow-up multivariate analyses) further suggests that these mechanisms are sensitive to the degree of training in establishing these stimulusresponse mappings and become unnecessary whenever the same instructions are repeated (Ramamoorthy \& Verguts, 2012). In this light, our results may reflect learning procedures in which verbal information is transformed into sublinguistic representations usable by premotor systems for behavior when no training has yet occurred (cf. Brass et al., 2017; Brass, Wenke, Spengler, \& Waszak, 2009 , for similar suggestions). The specific role of the rMFG-dPMC could then be the activation of a generic if-then rule format (hence, the higher voxel pattern consistency across different task sequences) that allows the formation of such sensorimotor representations regardless of task sequence. These representations can be considered similar to the procedural working memory format proposed by Oberauer (2009). Importantly, although our results show that rMFG-dPMC responds more consistently to novel SR trials than trained SR trials, this does not mean that rMFG-dPMC is not involved in trained SR instruction processing. Instead, our results could just as well indicate rMFG-dPMC's involvement in processing task-specific information represented in previously applied trials (see especially Figure 6). At this point, we can only conclude that the rMFG-dPMC seems to be more involved in processing novel SR than novel OC instructions (i.e., univariate findings), replicating previous results (e.g., Demanet et al., 2016), and seems to do so in a more generalizable manner than it does for novel OC and trained SR instructions (i.e., multivariate findings).

Interestingly, our findings and previous evidence for rMFC-dPMC activity during novel instruction implementation (Demanet et al., 2016; Ruge \& Wolfensteller, 2010; see also whole-brain analyses in Muhle-Karbe et al., 2017) suggest that this region's involvement in novel instruction implementation requires that instructions contain, at least in part, explicitly verbal information. In contrast, instructions conveyed in an exclusively nonverbal format have been shown to recruit the right VLPFC (Reverberi et al., 2012a, 2012b). The absence of the right VLPFC involvement in our present study, whether at the univariate or multivariate level, indicates that the neural mechanisms underlying instruction implementation should, to some extent, be differentiated based on the format in which instructions are conveyed. Several hypotheses exist regarding a possible role of the right LPFC areas in incorporating verbal and nonverbal 
information for higher-level behaviors (Putt et al., 2017; Stout \& Chaminade, 2012). In line with previous hypotheses (Brass et al., 2009), we speculate that these incorporation processes may intervene at an intermediate stage between the processing of purely verbal information (likely involving language areas of the left hemisphere-a possibility entertained by Hartstra et al., 2011) and a sensorimotor stage in which verbally conveyed stimulusresponse mappings have been transformed into concrete sensorimotor representations for impending action. Understanding the exact moment-to-moment transition of rule-guided behaviors from instructions to implementation, however, requires more complex imaging techniques apt to disentangle the various stages of brain signal progression during task execution (Bode \& Haynes, 2009; Sigala, Kusunoki, Nimmo-Smith, Gaffan, \& Duncan, 2008).

Reprint requests should be sent to Nicolas J. Bourguignon, Department of Experimental Psychology, Ghent University, Henri Dunantlaan 2, Ghent, Belgium, 9000, or via e-mail: nicolas. bourguignon@ugent.be.

\section{Note}

1. Demanet et al. (2016) initially chose to name this region the inferior frontal sulcus (IFS) based on its main activation peak. However, given the anatomical overlap with the area reported by Ruge and Wolfensteller (2010) and for the sake of consistency, we consider the nomenclature rMFG-dPMC more appropriate.

\section{REFERENCES}

Anderson, J. R. (1983). A spreading activation theory of memory. Journal of Verbal Learning and Verbal Behavior, 22, 261-295.

Bode, S., \& Haynes, J.-D. (2009). Decoding sequential stages of task preparation in the human brain. Neuroimage, 45 , 606-613.

Boettinger, C. A., \& D'Esposito, M. (2005). Frontal networks for learning and executing arbitrary stimulus-response associations. Journal of Neuroscience, 25, 2723-2732.

Braem, S., De Houwer, J., Demanet, J., Yuen, K. S. L., Kalisch, R., \& Brass, M. (2017). Pattern analyses reveal separate experience-based fear memories in the human right amygdala. Journal of Neuroscience, 37, 8116-8130.

Brass, M., Derrfuss, J., Forstmann, B., \& von Cramon, D. Y. (2005). The role of the inferior frontal junction area in cognitive control. Trends in Cognitive Sciences, 9, 314-316.

Brass, M., Liefooghe, B., Braem, S., \& De Houwer, J. (2017). Following new task instructions: Evidence for a dissociation between knowing and doing. Neuroscience and Biobehavioral Reviews, 81, 16-28.

Brass, M., Wenke, D., Spengler, S., \& Waszak, F. (2009). Neural correlates of overcoming interference from instructed and implemented stimulus-response associations. Journal of Neuroscience, 29, 1766-1772.

Cronbach, L. J. (1951). Coefficient alpha and the internal structure of tests. Psychometrika, 16, 297-334.

Deiber, M.-P., Wise, S. P., Honda, M., Catalan, M. J., Grafman, J., \& Hallett, M. (1997). Frontal and parietal networks for conditional motor learning: A positron emission tomography study. Journal of Neurophysiology, 78, 977-991.

Demanet, J., Liefooghe, B., Hartstra, E., Wenke, D., De Houwer, J., \& Brass, M. (2016). There is more into 'doing' than 'knowing': The function of the right inferior frontal sulcus is specific for implementing versus memorising verbal instructions. Neuroimage, 141, 350-356.

Derrfuss, J., Brass, M., \& von Cramon, D. Y. (2004). Cognitive control in the posterior frontolateral cortex: Evidence from common activations in task coordination, interference control, and working memory. Neuroimage, 23, 604-612.

Duncan, J. (2013). The structure of cognition: Attentional episodes in mind and brain. Neuron, 80, 35-50.

Duncan, J., Emslie, H., Williams, P., Johnson, R., \& Freer, C. (1996). Intelligence and the frontal lobe: The organization of goal-directed behavior. Cognitive Psychology, 30, 257-303.

Duncan, J., Hazel, E., Williams, P., Johnson, R., \& Freer, C. (1996). Intelligence and the frontal lobe: The organization of goal-directed behavior. Cognitive Psychology, 30, 257-303.

Duncan, J., Parr, A., Woolgar, A., Thompson, R., Bright, P., Cox, S., et al. (2008). Goal neglect and spearman's g: Competing parts of complex task. Journal of Experimental Psychology. General, 137, 131-148.

Everaert, T., Theeuwes, M., Liefooghe, B., \& De Houwer, J. (2014). Automatic motor activation by mere instruction. Cognitive, Affective, \& Behavioral Neuroscience, 14, 1300-1309.

Gilbert, S. J. (2011). Decoding the content of delayed intentions. Journal of Neuroscience, 31, 2888-2894.

Hartstra, E., Kühn, S., Verguts, T., \& Brass, M. (2011). The implementation of verbal instructions: An fMRI study. Human Brain Mapping, 32, 1811-1824.

Kriegeskorte, N., \& Bandettini, P. (2007). Analyzing for information, not activation, to exploit high-resolution fMRI. Neuroimage, 38, 649-662.

Kriegeskorte, N., Goebel, R., \& Bandettini, P. (2006). Information-based functional brain mapping. Proceedings of the National Academy of Sciences, U.S.A., 103, 3863-3868.

Liefooghe, B., De Houwer, J., \& Wenke, D. (2013). Instructionbased response activation depends on task preparation. Psychonomic Bulletin \& Review, 20, 481-487.

Liefooghe, B., Wenke, D., \& De Houwer, J. (2012). Instructionbased task-rule congruency effects. Journal of Experimental Psychology: Learning, Memory, and Cognition, 38, 1325-1335.

Luria, A. R. (1966). Higher cortical functions in man. London: Tavistock.

McDonald, R. P. (1999). Test theory: A unified treatment. Mahwah, NJ: Erlbaum.

Meiran, N., Liefooghe, B., \& De Houwer, J. (2017). Powerful instructions: Automaticity without practice. Current Directions in Psychological Science, 26, 509-514.

Meiran, N., Pereg, M., Kessler, Y., Cole, M. W., \& Braver, T. S. (2015). The power of instructions: Proactive configuration of stimulus-response translation. Journal of Experimental Psychology: Learning, Memory, and Cognition, 41, 768-786.

Morgan, T. J. H., Uomini, N. T., Rendell, L. E., Chouinard-Thuly, L., Street, S. E., Lewis, H. M., et al. (2015). Experimental evidence for the co-evolution of hominin tool-making teaching and language. Nature Communications, 6, 6029.

Muhle-Karbe, P. S., Duncan, J., De Baene, W., Mitchell, D. J., \& Brass, M. (2017). Neural coding for instruction-based task sets in human frontoparietal and visual cortex. Cerebral Cortex, 27, 1891-1905.

Norman, K. A., Polyn, S. M., Detre, G. J., \& Haxby, J. V. (2006). Beyond mind-reading: Multi-voxel pattern analysis of fMRI data. Trends in Cognitive Sciences, 10, 424-430. 
Oberauer, K. (2009). Design for a working memory. Psychology of Learning and Motivation, 51, 45-100.

Oldfield, R. C. (1971). The assessment and analysis of handedness: The Edinburgh inventory. Neuropsychologia, 9, 97-113.

Pochon, J.-B., Levy, R., Poline, J.-B., Crozier, S., Lehéricy, S., Pillon, B., et al. (2001). The role of dorsolateral prefrontal cortex in the preparation of forthcoming actions: An fMRI study. Cerebral Cortex, 11, 260-266.

Putt, S. S., Wijeakumar, S., Franciscus, R. G., \& Spencer, J. P. (2017). The functional brain networks that underlie Early Stone Age tool manufacture. Nature Human Behavior, 1, 0102.

Raichle, M. E., Fiez, J. A., Videen, T. O., MacLeod, A.-M. K., Pardo, J. V., Fox, P. T., et al. (1994). Practice-related changes in human brain functional anatomy during nonmotor learning. Cerebral Cortex, 4, 8-26.

Ramamoorthy, A., \& Verguts, T. (2012). Word and deed: A computational model of instruction following. Brain Research, 1439, 54-65.

Reverberi, C., Görgen, K., \& Haynes, J.-D. (2012a). Compositionality of rule representations in human prefrontal cortex. Cerebral Cortex, 22, 1237-1246.

Reverberi, C., Görgen, K., \& Haynes, J.-D. (2012b). Distributed representations of rule identity and rule order in human frontal cortex and striatum. Journal of Neuroscience, 32, $17420-17430$

Rowe, J. B., Toni, I., Josephs, O., Frackowiak, R. S. J., \& Passingham, R. E. (2000). The prefrontal cortex: Response selection or maintenance within working memory? Science, 288, 1656-1660.

Ruge, H., \& Wolfensteller, U. (2010). Rapid formation of pragmatic rule representations in the human brain during instruction-based learning. Cerebral Cortex, 20, 1656-1667.

Severens, E., Van Lommel, S., Ratinckx, E., \& Hartsuiker, R. J. (2005). Timed picture naming norms for 590 pictures in Dutch. Acta Psychologica, 119, 159-187.

Sigala, N., Kusunoki, M., Nimmo-Smith, I., Gaffan, D., \& Duncan, J. (2008). Hierarchical coding for sequential task events in the monkey prefrontal cortex. Proceedings of the National Academy of Sciences, U.S.A., 105, 11969-11974.

Stout, D., \& Chaminade, T. (2012). Stone tools, language and the brain in human evolution. Philosophical Transactions of the Royal Society of London, Series B: Biological Sciences, 367, 75-87.

Zach, N., Inbar, D., Grinvald, Y., Bergmann, H., \& Vaadia, E. (2008). Emergence of novel representations in primary motor cortex and premotor neurons during associative learning. Journal of Neuroscience, 28, 9545-9556. 